\title{
$q$-Deformed Fock spaces and modular representations of spin symmetric groups
}

\author{
Bernard Leclerc $†$ and Jean-Yves Thibon $\ddagger$ \\ †Département de Mathématiques, Université de Caen, Esplanade de la Paix, BP 5186, \\ 14032 Caen cedex, France \\ $\ddagger$ Institut Gaspard Monge, Université de Marne-la-Vallée, 2 rue de la Butte-Verte, 93166 \\ Noisy-le-Grand cedex, France
}

\begin{abstract}
We use the Fock space representation of the quantum affine algebra of type $A_{2 n}^{(2)}$ to obtain a description of the global crystal basis of its basic level 1 module. We formulate a conjecture relating this basis to decomposition matrices of spin symmetric groups in characteristic $2 n+1$.
\end{abstract}

\section{Introduction}

The Fock space representation of the quantum affine algebra $U_{q}\left(\widehat{s} l_{n}\right)=U_{q}\left(A_{n-1}^{(1)}\right)$ was constructed by Hayashi [16]. A combinatorial version of this construction was then used by Misra and Miwa [29] to describe Kashiwara's crystal basis of the basic representation $V\left(\Lambda_{0}\right)$. This made it possible to compute the global crystal basis of $V\left(\Lambda_{0}\right)$ [26]. Then, it was conjectured that the degree $m$ part of the transition matrices giving the coefficients of the global basis on the natural basis of the Fock space were $q$-analogues of the decomposition matrices of the type $A$ Hecke algebras $H_{m}$ at an $n$th root of unity [26]. According to a conjecture of James [17], these should coincide, for $n$ prime and large enough, with the decomposition matrices of symmetric groups $\mathrm{S}_{m}$ over a field of characteristic $n$. The conjecture of [26] has been proved by Ariki [3], and by Grojnowski [15] using the results of [14].

There is another approach to the calculation of decomposition matrices of type $A$ Hecke algebras, relying upon Soergel's results on tilting modules for quantum groups at roots of unity [36, 37]. This approach also leads to $q$-analogues of decomposition numbers expressed in terms of Kazhdan-Lusztig polynomials. It seems that these $q$ analogues are the same as those of [26] but there is no proof of this coincidence. In fact, the relationship between the two approaches is still unclear.

The results of 26, 3, 15] have been applied recently by Foda et al. [12] to determine which simple $H_{m}$-modules remain simple after restriction to $H_{m-1}$ and to show that this 
problem is equivalent to the decomposition of a tensor product of level $1 A_{n-1}^{(1)}$-modules. This provided an explanation for an intriguing correspondence previously observed in [13] between a class of RSOS models and modular representations of symmetric groups. Another description of the $U_{q}\left(A_{n-1}^{(1)}\right)$-Fock space, as a deformation of the infinite wedge realization of the fermionic Fock space, was obtained by Stern [38]. In [25], the $q$-bosons needed for the decomposition of the Fock space into irreducible $U_{q}\left(A_{n-1}^{(1)}\right)$ modules were introduced. This construction was used in 27 to give a combinatorial formula for the highest weight vectors, and in [28] to define a canonical basis of the whole Fock space which was conjectured to yield the decomposition matrices of $q$-Schur algebras at roots of unity. Moreover, strong support in favor of this conjecture was obtained by establishing its compatibility with a version of the Steinberg tensor product theorem proved by James in this context [17, 28].

Recently, the theory of perfect crystals [21, 22] allowed Kashiwara et al. 24] to define a general notion of $q$-Fock space, extending the results of [25] to several series of affine algebras. Their results apply in particular to the twisted affine algebra of type $A_{2 n}^{(2)}$, which is the case considered in this note.

It has been noticed by Nakajima and Yamada [33] that the combinatorics of the basic representation $V\left(\Lambda_{n}\right)$ of $A_{2 n}^{(2)}$ was similar to the one encountered in the $(2 n+1)$ modular representation theory of the spin symmetric groups $\widehat{\mathrm{S}}_{m}$ by Morris [30 as early as 1965. This can be explained to a certain extent by observing that the $(r, \bar{r})$-inducing operators of Morris and Yaseen [32] coincide with the Chevalley lowering operators of the Fock space representation of $A_{2 n}^{(2)}$. This provides a further example of the phenomenon observed in [26] in the case of symmetric groups and $A_{n-1}^{(1)}$-algebras.

In this note, we give the analogues for $U_{q}\left(A_{2 n}^{(2)}\right)$ of the results of [26]. Using the level $1 q$-Fock spaces of [24], we describe an algorithm for computing the canonical basis of the basic representation $V\left(\Lambda_{n}\right)$, which allows us to prove that this basis is in the $\mathbf{Z}[q]$-lattice spanned by the natural basis of the $q$-Fock space, and that the transition matrices have an upper triangle of zeros (Theorem 4.1).

We conjecture that the specialization $q=1$ gives, up to splitting of rows and columns for pairs of associate characters, and for sufficiently large primes $p=2 n+1$, the decomposition matrices of spin symmetric groups. However, the reduction $q=1$ is more tricky than in the $A_{n-1}^{(1)}$ case. Indeed, the $q$-Fock space of $A_{2 n}^{(2)}$ is strictly larger than the classical one, and one has to factor out the null space of a certain quadratic form [24] to recover the usual description.

The missing ingredient in the spin case when we compare it to [26] is that, since the spin symmetric groups are not Coxeter groups, there is no standard way of associating to them a Hecke algebra, and this is an important obstruction for proving our conjecture. What we can actually prove is that all self-associate projective characters of $\widehat{\mathrm{S}}_{m}$ are linear combinations of characters obtained from smaller groups by a sequence of $(r, \bar{r})$ - 
inductions (Theorem 6.1). This proof is constructive in the sense that the intermediate basis $\{A(\mu)\}$ of our algorithm for the canonical basis, suitably specialized at $q=1$, is a basis for the space spanned by such characters.

This should have implications on the way of labelling the irreducible modular spin representations of $\widehat{S}_{m}$. Up to now, a coherent labelling scheme has been found only for $p=3$ [8] and $p=5$ [1]. The case $p \geq 7$ led to formidable difficulties. To overcome this problem, we propose to use the labels of the crystal graph of $V\left(\Lambda_{n}\right)$, which may contain partitions with repeated parts not arising in the representation theory of $\widehat{S}_{m}$, and corresponding to ghost vectors of the $q$-Fock space at $q=1$.

\section{The Fock space representation of $U_{q}\left(A_{2 n}^{(2)}\right)$}

The Fock space representation of the affine Lie algebra $A_{2 n}^{(2)}$ can be constructed by means of its embedding in $b_{\infty}=\widehat{g o}_{\infty}$, the completed infinite rank affine Lie algebra of type $B$ [10, 11].

The (bosonic) Fock space of type $B$ is the polynomial algebra $\mathcal{F}=\mathbf{C}\left[p_{2 j+1}, j \geq 0\right]$ in an infinite number of generators $p_{2 j+1}$ of odd degree $2 j+1$. If one identifies $p_{k}$ with the power sum symmetric function $p_{k}=\sum_{i} x_{i}^{k}$ in some infinite set of variables, the natural basis of weight vectors for $b_{\infty}$ is given by Schur's $P$-functions $P_{\lambda}$ (where $\lambda$ runs over the set DP of partitions into distinct parts) [10, 40, 19.

The Chevalley generators $e_{i}^{\infty}, f_{i}^{\infty}(i \geq 0)$ of $b_{\infty}$ act on $P_{\lambda}$ by

$$
e_{i}^{\infty} P_{\lambda}=P_{\mu}, \quad f_{i}^{\infty} P_{\lambda}=P_{\nu}
$$

where $\mu$ (resp. $\nu$ ) is obtained from $\lambda$ by replacing its part $i+1$ by $i$ (resp. its part $i$ by $i+1$ ), the result being 0 if $i+1$ (resp. $i$ ) is not a part of $\lambda$. Also, it is understood that $P_{\mu}=0$ as soon as $\mu$ has a multiple part. For example, $f_{0}^{\infty} P_{32}=P_{321}, f_{3}^{\infty} P_{32}=P_{42}$, $e_{1}^{\infty} P_{32}=P_{31}$ and $e_{2}^{\infty} P_{32}=P_{22}=0$.

Let $h=2 n+1$. The Chevalley generators $e_{i}, f_{i}$ of $A_{2 n}^{(2)}$ will be realized as

$$
\begin{aligned}
f_{i} & =\sum_{j \equiv n \pm i} f_{j}^{\infty} \quad(i=0, \ldots, n), \\
e_{i} & =\sum_{j \equiv n \pm i} e_{j}^{\infty} \quad(i=0, \ldots, n-1), \quad e_{n}=e_{0}^{\infty}+2 \sum_{\substack{j>0 \\
j \equiv 0,-1}} e_{j}^{\infty},
\end{aligned}
$$

where all congruences are taken modulo $h$. Let $A_{2 n}^{(2) \prime}$ be the derived algebra of $A_{2 n}^{(2)}$ (obtained by omitting the degree operator $d$ ). The action of $A_{2 n}^{(2) \prime}$ on $\mathcal{F}$ is centralized by the Heisenberg algebra generated by the operators $\frac{\partial}{\partial p_{h s}}$ and $p_{h s}$ for odd $s \geq 1$. This implies that the Fock space decomposes under $A_{2 n}^{(2)}$ as

$$
\mathcal{F}=\bigoplus_{k \geq 0} V\left(\Lambda_{n}-k \delta\right)^{\oplus p^{*}(k)}
$$


where $p^{*}(k)$ is the number of partitions of $k$ into odd parts. In particular, the subrepresentation generated by the vacuum vector $|0\rangle=P_{0}=1$ is the basic representation $V\left(\Lambda_{n}\right)$ of $A_{2 n}^{(2)}$, and its principally specialized character is [20]

$$
\operatorname{ch}_{t} V\left(\Lambda_{n}\right)=\sum_{m \geq 0} \operatorname{dim} V\left(\Lambda_{n}\right)_{m} t^{m}=\prod_{\substack{i \text { odd } \\ i \neq 0 \text { mod }}} \frac{1}{1-t^{i}}
$$

The $q$-deformation of this situation has been discovered by Kashiwara et al. 24. Contrary to the case of $A_{n-1}^{(1)}$, the $q$-Fock space is strictly larger than the classical one. We recall here briefly their construction, referring to [24 for details and notation.

Let $\mathrm{DP}_{h}(m)$ be the set of partitions $\lambda=\left(1^{m_{1}} 2^{m_{2}} \ldots r^{m_{r}}\right)$ of $m$ for which $m_{i} \leq 1$ when $i \not \equiv 0 \bmod h$. For example, $\mathrm{DP}_{3}(7)=\{(7),(61),(52),(43),(421),(331)\}$. Set $\mathrm{DP}_{h}=\bigcup_{m} \mathrm{DP}_{h}(m)$. Then, the $q$-Fock space of type $A_{2 n}^{(2)}$ is

$$
\mathcal{F}_{q}=\bigoplus_{\lambda \in \mathrm{DP}_{h}} \mathbf{Q}(q)|\lambda\rangle
$$

where for $\lambda=\left(\lambda_{1}, \ldots, \lambda_{r}\right),|\lambda\rangle$ denotes the infinite $q$-wedge product

$$
|\lambda\rangle=u_{\lambda}=u_{\lambda_{1}} \wedge_{q} u_{\lambda_{2}} \wedge_{q} \cdots \wedge_{q} u_{\lambda_{r}} \wedge_{q} u_{0} \wedge_{q} u_{0} \wedge_{q} \cdots
$$

of basis vectors $u_{i}$ of the representation $V_{\text {aff }}$. The quantum affine algebra $U_{q}\left(A_{2 n}^{(2)}\right)$ acts on $V_{\text {aff }}=\bigoplus_{i \in \mathbf{Z}} \mathbf{Q}(q) u_{i}$ by

$$
\begin{aligned}
& f_{i} u_{j}=\left\{\begin{array}{ll}
u_{j+1} & \text { if } j \equiv n \pm i \bmod h \\
0 & \text { otherwise }
\end{array} \quad(i=0, \ldots, n-1)\right. \\
& f_{n} u_{j}= \begin{cases}u_{j+1} & \text { if } j \equiv-1 \bmod h \\
\left(q+q^{-1}\right) u_{j+1} & \text { if } j \equiv 0 \bmod h \\
0 & \text { otherwise }\end{cases} \\
& e_{i} u_{j}=\left\{\begin{array}{ll}
u_{j-1} & \text { if } j \equiv n+1 \pm i \bmod h \\
0 & \text { otherwise }
\end{array} \quad \text { if } j \equiv 1 \bmod h\right. \\
& e_{n} u_{j}= \begin{cases}u_{j-1} & \text { if } j \equiv 0 \bmod h \\
\left(q+q^{-1}\right) u_{j-1} & \text { otherwise } \\
0 & \text { if } j \equiv n \bmod h\end{cases} \\
& t_{0} u_{j}=\left\{\begin{array}{ll}
q^{4} u_{j} & \text { if } j \equiv n+1 \bmod h \\
q^{-4} u_{j} & \text { otherwise } \\
u_{j} & \text { if } j \equiv n \pm i \bmod h
\end{array} \quad(i=1, \ldots, n-1)\right. \\
& t_{i} u_{j}= \begin{cases}q^{2} u_{j} & \text { if } j \equiv n+1 \pm i \bmod h \\
q^{-2} u_{j} & \text { otherwise } \\
u_{j} & \end{cases}
\end{aligned}
$$




$$
t_{n} u_{j}= \begin{cases}q^{2} u_{j} & \text { if } j \equiv-1 \bmod h \\ q^{-2} u_{j} & \text { if } j \equiv 1 \bmod h \\ u_{j} & \text { otherwise }\end{cases}
$$

The only commutation rules we will need to describe the action of $e_{i}$ and $f_{i}$ on $\mathcal{F}_{q}$ are:

$$
\begin{aligned}
& u_{j} \wedge_{q} u_{j}=0 \text { if } j \not \equiv 0 \bmod h \\
& u_{j} \wedge_{q} u_{j+1}=-q^{2} u_{j+1} \wedge_{q} u_{j} \text { if } j \equiv 0,-1 \bmod h .
\end{aligned}
$$

The action on the vacuum vector $|0\rangle=u_{0} \wedge_{q} u_{0} \wedge_{q} \cdots$ is given by

$$
e_{i}|0\rangle=0, \quad f_{i}|0\rangle=\delta_{\text {in }}|1\rangle, \quad t_{i}|0\rangle=q^{\delta_{i n}}|0\rangle,
$$

and on a $q$-wedge $|\lambda\rangle=u_{\lambda_{1}} \wedge_{q} \cdots \wedge_{q} u_{\lambda_{r}} \wedge_{q}|0\rangle$,

$$
\begin{aligned}
f_{i}|\lambda\rangle= & f_{i} u_{\lambda_{1}} \wedge_{q} t_{i} u_{\lambda_{2}} \wedge_{q} \cdots t_{i} u_{\lambda_{r}} \wedge_{q} t_{i}|0\rangle \\
& +u_{\lambda_{1}} \wedge_{q} f_{i} u_{\lambda_{2}} \wedge_{q} \cdots t_{i} u_{\lambda_{r}} \wedge_{q} t_{i}|0\rangle \\
& +\cdots+u_{\lambda_{1}} \wedge_{q} u_{\lambda_{2}} \wedge_{q} \cdots u_{\lambda_{r}} \wedge_{q} f_{i}|0\rangle \\
e_{i}|\lambda\rangle= & t_{i}^{-1} u_{\lambda_{1}} \wedge_{q} t_{i}^{-1} u_{\lambda_{2}} \wedge_{q} \cdots t_{i}^{-1} u_{\lambda_{r}} \wedge_{q} e_{i}|0\rangle \\
& +t_{i}^{-1} u_{\lambda_{1}} \wedge_{q} t_{i}^{-1} u_{\lambda_{2}} \wedge_{q} \cdots e_{i} u_{\lambda_{r}} \wedge_{q}|0\rangle \\
& +\cdots+e_{i} u_{\lambda_{1}} \wedge_{q} u_{\lambda_{2}} \wedge_{q} \cdots u_{\lambda_{r}} \wedge_{q}|0\rangle \\
& \\
t_{i}|\lambda\rangle= & t_{i} u_{\lambda_{1}} \wedge_{q} t_{i} u_{\lambda_{2}} \wedge_{q} \cdots \wedge_{q} t_{i} u_{\lambda_{r}} \wedge_{q} t_{i}|0\rangle .
\end{aligned}
$$

For example, with $n=2$, one has

$$
f_{2}|542\rangle=\left(q^{4}+q^{2}\right)|642\rangle+q|552\rangle+|5421\rangle,
$$

and

$$
f_{2}|552\rangle=\left(q^{2}+1\right)(|652\rangle+|562\rangle)+|5521\rangle=\left(1-q^{4}\right)|652\rangle+|5521\rangle,
$$

the last equality resulting from (15).

It is proved in 24] that $\mathcal{F}_{q}$ is an integrable highest weight $U_{q}\left(A_{2 n}^{(2)}\right)$-module whose decomposition into irreducible components, obtained by means of $q$-bosons, is

$$
\mathcal{F}_{q}=\bigoplus_{k \geq 0} V\left(\Lambda_{n}-k \delta\right)^{\oplus p(k)}
$$

where $p(k)$ is now the number of all partitions of $k$ (compare (4)). Thus, the submodule $U_{q}\left(A_{2 n}^{(2)}\right)|0\rangle$ is a realization of the basic representation $V\left(\Lambda_{n}\right)$. 


\section{The crystal graph of the $q$-Fock space}

The first step in computing the global basis of $V\left(\Lambda_{n}\right) \subset \mathcal{F}_{q}$ is to determine the crystal basis of $\mathcal{F}_{q}$ whose description follows from [24, 21, 22]. Let $A$ denote the subring of $\mathbf{Q}(q)$ consisting of rational functions without pole at $q=0$. The crystal lattice of $\mathcal{F}_{q}$ is $L=\bigoplus_{\lambda \in \mathrm{DP}_{h}} A|\lambda\rangle$, and the crystal basis of the $\mathbf{Q}$-vector space $L / q L$ is $B=\left\{|\lambda\rangle \bmod q L, \lambda \in \mathrm{DP}_{h}\right\}$. We shall write $\lambda$ instead of $|\lambda\rangle \bmod q L$.

The Kashiwara operators $\tilde{f}_{i}$ act on $B$ in a simple way recorded on the crystal graph $\Gamma\left(\mathcal{F}_{q}\right)$. To describe this graph, one starts with the crystal graph $\Gamma\left(V_{\text {aff }}\right)$ of $V_{\text {aff. }}$ This is the graph with vertices $j \in \mathbf{Z}$, whose arrows labelled by $i \in\{0,1, \ldots, n\}$ are given, for $i \neq n$, by

$$
j \stackrel{i}{\longrightarrow} j+1 \Longleftrightarrow j \equiv n \pm i \bmod h
$$

and for $i=n$ by

$$
j \stackrel{n}{\longrightarrow} j+1 \Longleftrightarrow j \equiv-1,0 \bmod h .
$$

Thus for $n=2$ this graph is

$$
\text { ‥ } \stackrel{1}{\longrightarrow}-1 \stackrel{2}{\longrightarrow} 0 \stackrel{2}{\longrightarrow} 1 \stackrel{1}{\longrightarrow} 2 \stackrel{0}{\longrightarrow} 3 \stackrel{1}{\longrightarrow} 4 \stackrel{2}{\longrightarrow} 5 \stackrel{2}{\longrightarrow} 6 \stackrel{1}{\longrightarrow} 7 \stackrel{0}{\longrightarrow} \cdots
$$

The graph $\Gamma\left(\mathcal{F}_{q}\right)$ is obtained inductively from $\Gamma\left(V_{\text {aff }}\right)$ using the following rules. Let $\lambda=\left(\lambda_{1}, \ldots, \lambda_{r}\right) \in B$, and write $\lambda=\left(\lambda_{1}, \lambda^{*}\right)$ where $\lambda^{*}=\left(\lambda_{2}, \ldots, \lambda_{r}\right)$. Then one has $\tilde{f}_{i}(0)=\delta_{i n}(1), \varphi_{i}(0)=\delta_{i n}$, and

$$
\tilde{f}_{i} \lambda=\left\{\begin{array}{l}
\left(\tilde{f}_{i} \lambda_{1}, \lambda^{*}\right) \text { if } \varepsilon_{i}\left(\lambda_{1}\right) \geq \varphi_{i}\left(\lambda^{*}\right), \\
\left(\lambda_{1}, \tilde{f}_{i} \lambda^{*}\right) \text { if } \varepsilon_{i}\left(\lambda_{1}\right)<\varphi_{i}\left(\lambda^{*}\right) .
\end{array}\right.
$$

Here, $\varepsilon_{i}\left(\lambda_{1}\right)$ means the distance in $\Gamma\left(V_{\text {aff }}\right)$ from $\lambda_{1}$ to the origin of its $i$-string, and $\varphi_{i}\left(\lambda^{*}\right)$ means the distance in $\Gamma\left(\mathcal{F}_{q}\right)$ from $\lambda^{*}$ to the end of its $i$-string.

Thus for $n=1$ one computes successively the following 1-strings of $\Gamma\left(\mathcal{F}_{q}\right)$

$$
\begin{aligned}
& (0) \stackrel{1}{\longrightarrow}(1) \\
& (2)=(2,0) \stackrel{1}{\longrightarrow}(2,1) \stackrel{1}{\longrightarrow}(3,1) \stackrel{1}{\longrightarrow}(4,1) \\
& (3,2)=(3,2,0) \stackrel{1}{\longrightarrow}(3,2,1) \stackrel{1}{\longrightarrow}(3,3,1) \stackrel{1}{\longrightarrow}(4,3,1)
\end{aligned}
$$

from which one deduces that $\tilde{f}_{1}(3,3,1)=(4,3,1)$ and $\varphi_{1}(3,3,1)=1$.

The first layers of the crystal $\Gamma\left(\mathcal{F}_{q}\right)$ for $n=1$ are shown in Fig. 1. One can observe that the decomposition of $\Gamma\left(\mathcal{F}_{q}\right)$ into connected components reflects the decomposition (20) of $\mathcal{F}_{q}$ into simple modules. More precisely, the connected components of $\Gamma\left(\mathcal{F}_{q}\right)$ are all isomorphic as colored graphs to the component $\Gamma\left(\Lambda_{n}\right)$ containing the empty partition. Their highest vertices are the partitions $\nu$ whose parts are all divisible by $h$. 



Figure 1. The graph $\Gamma\left(\mathcal{F}_{q}\right)$ for $A_{2}^{(2)}$ up to degree 7

This follows from the fact, easily deduced from the rules we have just explained, that if $\nu=h \mu=\left(h \mu_{1}, \ldots, h \mu_{r}\right)$ is such a partition, then the map

$$
\lambda \mapsto \lambda+\nu=\left(\lambda_{1}+h \mu_{1}, \lambda_{2}+h \mu_{2}, \ldots\right)
$$

is a bijection from $\Gamma\left(\Lambda_{n}\right)$ onto the connected component of $\Gamma\left(\mathcal{F}_{q}\right)$ containing $\nu$, and this bijection commutes with the operators $\tilde{e}_{i}$ and $\tilde{f}_{i}$. This implies that the vertices of $\Gamma\left(\Lambda_{n}\right)$ are the partitions $\lambda=\left(\lambda_{1}, \ldots, \lambda_{r}, 0\right) \in \mathrm{DP}_{h}$ such that for $i=1,2, \ldots, r$, one has $\lambda_{i}-\lambda_{i+1} \leq h$ and $\lambda_{i}-\lambda_{i+1}<h$ if $\lambda_{i} \equiv 0 \bmod h$. We shall call a partition that satisfies these conditions $h$-regular. The set of $h$-regular partitions of $m$ will be denoted by $\mathrm{DPR}_{h}(m)$, and we shall write $\mathrm{DPR}_{h}=\cup_{m} \operatorname{DPR}_{h}(m)$.

For example,

$$
\operatorname{DPR}_{3}(10)=\{(3331),(4321),(532),(541)\} .
$$

\section{The canonical basis of $V\left(\Lambda_{n}\right)$}

In this section, we describe an algorithm for computing the canonical basis (global lower crystal basis) of the basic representation $V\left(\Lambda_{n}\right)=U_{q}\left(A_{2 n}^{(2)}\right)|0\rangle$ in terms of the natural 
basis $|\lambda\rangle$ of the $q$-Fock space. To characterize the canonical basis, we need the following notations

$$
q_{i}=\left\{\begin{array}{ll}
q & \text { if } i=n \\
q^{2} & \text { if } 1 \leq i<n \\
q^{4} & \text { if } i=0
\end{array} \quad t_{i}= \begin{cases}q^{h_{n}} & \text { if } i=n \\
q^{2 h_{i}} & \text { if } 1 \leq i<n \\
q^{4 h_{0}} & \text { if } i=0\end{cases}\right.
$$

and

$$
[k]_{i}=\frac{q_{i}^{k}-q_{i}^{-k}}{q_{i}-q_{i}^{-1}}, \quad[k]_{i} !=[k]_{i}[k-1]_{i} \cdots[1]_{i} .
$$

The $q$-divided powers of the Chevalley generators are defined by

$$
e_{i}^{(k)}=\frac{e_{i}^{k}}{[k]_{i} !}, \quad f_{i}^{(k)}=\frac{f_{i}^{k}}{[k]_{i} !} .
$$

The canonical basis is defined in terms of an involution $v \mapsto \bar{v}$ of $V\left(\Lambda_{n}\right)$. Let $x \mapsto \bar{x}$ be the ring automorphism of $U_{q}\left(A_{2 n}^{(2)}\right)$ such that $\bar{q}=q^{-1}, \overline{q^{h}}=q^{-h}$ for $h$ in the Cartan subalgebra of $A_{2 n}^{(2)}$, and $\overline{e_{i}}=e_{i}, \overline{f_{i}}=f_{i}$. Then, for $v=x|0\rangle \in V\left(\Lambda_{n}\right)$, define $\bar{v}=\bar{x}|0\rangle$.

We denote by $U_{\mathbf{Q}}^{-}$the sub-Q $\left[q, q^{-1}\right]$-algebra of $U_{q}\left(A_{2 n}^{(2)}\right)$ generated by the $f_{i}^{(k)}$ and set $V_{\mathbf{Q}}\left(\Lambda_{n}\right)=U_{\mathbf{Q}}^{-}|0\rangle$. Then, as shown by Kashiwara [23], there exists a unique $\mathbf{Q}\left[q, q^{-1}\right]$ basis $\left\{G(\mu), \mu \in \mathrm{DPR}_{h}\right\}$ of $V_{\mathbf{Q}}\left(\Lambda_{n}\right)$, such that

(G1) $G(\mu) \equiv|\mu\rangle \bmod q L$

(G2) $\overline{G(\mu)}=G(\mu)$.

To compute $G(\mu)$, we follow the same strategy as in [26]. We first introduce an auxiliary basis $A(\mu)$ satisfying (G2), from which we manage to construct combinations satisfying also (G1). More precisely, let $\mathcal{F}_{q}^{m}$ be the subspace of $\mathcal{F}_{q}$ spanned by $|\lambda\rangle$ for $\lambda \in \mathrm{DP}_{h}(m)$ and set $V\left(\Lambda_{n}\right)_{m}=\mathcal{F}_{q}^{m} \cap V\left(\Lambda_{n}\right)$. Denote by $\unlhd$ the natural order on partitions. Then, the auxiliary basis will satisfy

(A0) $\left\{A(\mu), \mu \in \mathrm{DPR}_{h}(m)\right\}$ is a $\mathbf{Q}\left[q, q^{-1}\right]$-basis of $V_{\mathbf{Q}}\left(\Lambda_{n}\right)_{m}$,

(A1) $A(\mu)=\sum_{\lambda} a_{\lambda \mu}(q)|\lambda\rangle$, where $a_{\lambda \mu}(q)=0$ unless $\lambda \unrhd \mu, a_{\mu \mu}(q)=1$ and $a_{\lambda \mu}(q) \in \mathbf{Z}\left[q, q^{-1}\right]$

(A2) $\overline{A(\mu)}=A(\mu)$.

The basis $A(\mu)$ is obtained by applying monomials in the $f_{i}^{(k)}$ to the highest weight vector, that is, $A(\mu)$ is of the form

$$
A(\mu)=f_{r_{s}}^{\left(k_{s}\right)} f_{r_{s-1}}^{\left(k_{s-1}\right)} \cdots f_{r_{1}}^{\left(k_{1}\right)}|0\rangle
$$

so that (A2) is satisfied.

The two sequences $\left(r_{1}, \ldots, r_{s}\right)$ and $\left(k_{1}, \ldots, k_{s}\right)$ are, as in 26], obtained by peeling off the $A_{2 n}^{(2)}$-ladders of the partition $\mu$, which are defined as follows. We first fill the cells 
of the Young diagram $Y$ of $\mu$ with integers (called residues), constant in each column of $Y$. If $j \equiv n \pm i \bmod h(0 \leq i \leq n)$, the numbers filling the $j$-th column of $Y$ will be equal to $i$. A ladder of $\mu$ is then a sequence of cells with the same residue, located in consecutive rows at horizontal distance $h$, except when the residue is $n$, in which case two consecutive $n$-cells in a row belong also to the same ladder. For example, with $n=3$ and $\mu=(11,7,7,4)$, one finds 22 ladders (indicated by subscripts), the longest one being the 7 th, containing three 3 -cells:

$$
\begin{array}{|c|c|c|c|c|c|c|c|c|c|c|}
\hline 3_{19} & 2_{20} & 1_{21} & 0_{22} & \multicolumn{7}{|c|}{\mid} \\
\hline 3_{13} & 2_{14} & 1_{15} & 0_{16} & 1_{17} & 2_{18} & 3_{19} \\
\hline 3_{7} & 2_{8} & 1_{9} & 0_{10} & 1_{11} & 2_{12} & 3_{13} & \multicolumn{3}{|c|}{} \\
\hline 3_{1} & 2_{2} & 1_{3} & 0_{4} & 1_{5} & 2_{6} & 3_{7} & 3_{7} & 2_{8} & 1_{9} & 0_{10} \\
\hline
\end{array}
$$

Note that this definition of ladders agrees with that of [8] for $n=1$, but differs from that of [1] for $n=2$.

Then, in (25), $s$ is the number of ladders, $r_{i}$ the residue of the $i$ th ladder, and $k_{i}$ the number of its cells. Thus, proceeding with our example,

$A(11,7,7,4)=f_{0} f_{1} f_{2} f_{3}^{(2)} f_{2} f_{1} f_{0} f_{1} f_{2} f_{3}^{(2)} f_{2} f_{1} f_{0}^{(2)} f_{1}^{(2)} f_{2}^{(2)} f_{3}^{(3)} f_{2} f_{1} f_{0} f_{1} f_{2} f_{3}|0\rangle$.

The proof of $(\mathrm{A} 0)$ and $(\mathrm{A} 1)$ can be readily adapted from [26]. In particular, (A1) follows from the fact that a partition $\lambda$ belongs to $\mathrm{DPR}_{h}$ if and only if all cells of a given ladder intersecting $\lambda$ occupy the highest possible positions on this ladder.

Another choice of an intermediate basis, more efficient for practical computations, would be to use inductively the vectors $G(\nu)$ already computed and to set $A(\mu)=$ $f_{r_{s}}^{\left(k_{s}\right)} G(\nu)$, where $\nu$ is the partition obtained from $\mu$ by removing its outer ladder.

Define now the coefficients $b_{\nu \mu}(q)$ by

$$
G(\mu)=\sum_{\nu} b_{\nu \mu}(q) A(\nu)
$$

Still following [26], one can check that $b_{\nu \mu}(q)=0$ unless $\nu \geq \mu$, where $\geq$ denote the lexicographic ordering on partitions, and that $b_{\mu \mu}(q)=1$. Therefore, one can apply the triangular process of [26] as follows.

Let $\mu^{(1)}<\mu^{(2)}<\ldots<\mu^{(t)}$ be the set $\operatorname{DPR}_{h}(m)$ sorted in lexicographic order, so that $A\left(\mu^{(t)}\right)=G\left(\mu^{(t)}\right)$. Suppose that the expansion on the basis $|\lambda\rangle$ of $G\left(\mu^{(i+1)}\right), \ldots, G\left(\mu^{(t)}\right)$ has already been calculated. Then,

$$
G\left(\mu^{(i)}\right)=A\left(\mu^{(i)}\right)-\gamma_{i+1}(q) G\left(\mu^{(i+1)}\right)-\cdots-\gamma_{t}(q) G\left(\mu^{(t)}\right),
$$

where the coefficients are determined by the conditions

$$
\gamma_{s}\left(q^{-1}\right)=\gamma_{s}(q), \quad G\left(\mu^{(i)}\right) \equiv\left|\mu^{(i)}\right\rangle \bmod q L .
$$


Thus, for $n=1$, the first partition for which $A(\mu) \neq G(\mu)$ is $\mu=(3321)$ and

$$
\begin{aligned}
A(3321)= & |3321\rangle+q|333\rangle+\left(q^{2}-q^{6}\right)|432\rangle+\left(1+2 q^{2}\right)|531\rangle+\left(q^{2}+q^{4}\right)|54\rangle \\
& +\left(2 q^{2}+q^{4}\right)|621\rangle+2 q^{3}|63\rangle+\left(q^{4}+q^{6}\right)|72\rangle+q^{4}|81\rangle+q^{5}|9\rangle
\end{aligned}
$$

Indeed, $A(3321) \equiv|3321\rangle+|531\rangle \bmod q L$. On the other hand, $A(531)=|531\rangle+$ $q^{2}|54\rangle+q^{2}|621\rangle+q^{3}|63\rangle+q^{6}|72\rangle$ is equal to $G(531)$, and one finds by subtracting this from $A(3321)$ that

$$
\begin{aligned}
G(3321)= & |3321\rangle+q|333\rangle+\left(q^{2}-q^{6}\right)|432\rangle+2 q^{2}|531\rangle+q^{4}|54\rangle \\
& +\left(q^{2}+q^{4}\right)|621\rangle+q^{3}|63\rangle+q^{4}|72\rangle+q^{4}|81\rangle+q^{5}|9\rangle .
\end{aligned}
$$

Since $A(432)=|432\rangle+q^{4}|531\rangle+q^{2}|72\rangle+q^{6}|81\rangle$ satisfies (G1) and (G2), it has to be equal to $G(432)$, which completes the determination of the canonical basis for $m=9$. For $m=10$, the results are displayed as the columns of Table 11.

Table 1. The canonical basis for $n=1$ and $m=10$.

\begin{tabular}{lllll}
\hline & $(3331)$ & $(4321)$ & $(532)$ & $(541)$ \\
\hline$(3331)$ & 1 & 0 & 0 & 0 \\
$(4321)$ & $q-q^{5}$ & 1 & 0 & 0 \\
$(433)$ & $q^{2}$ & $q$ & 0 & 0 \\
$(532)$ & 0 & 0 & 1 & 0 \\
$(541)$ & $q+q^{3}$ & $q^{2}+q^{4}$ & 0 & 1 \\
$(631)$ & $2 q^{2}$ & $q^{3}$ & 0 & $q$ \\
$(64)$ & $q^{4}$ & 0 & 0 & $q^{3}$ \\
$(721)$ & $q^{3}+q^{5}$ & $q^{2}$ & 0 & $q^{4}$ \\
$(73)$ & $q^{4}$ & $q^{3}$ & 0 & $q^{5}$ \\
$(82)$ & 0 & 0 & $q^{2}$ & 0 \\
$(91)$ & $q^{4}$ & $q^{5}$ & 0 & 0 \\
$(10)$ & $q^{6}$ & 0 & 0 & 0 \\
\hline
\end{tabular}

In the Fock space representation of $A_{n-1}^{(1)}$, the weight of a basis vector $|\lambda\rangle$ is determined by the $n$-core of the partition $\lambda$ (and its degree) [4, 26]. There is a similar result of Nakajima and Yamada [33] for $A_{2 n}^{(2)}$, in terms of the notion of $\bar{h}$-core of a strict partition introduced by Morris [30] in the context of the modular representation theory of spin symmetric groups.

One way to see this is to use a theorem of [31] according to which $\lambda, \mu \in \operatorname{DP}(m)$ have the same $\bar{h}$-core if and only if they have, for each $i$, the same number $n_{i}$ of nodes of residue $i$. On the other hand, it follows from the implementation of the Chevalley generators that $|\lambda\rangle$ has $A_{2 n}^{(2)}$-weight $\Lambda_{n}-\sum_{0 \leq i \leq n} n_{i} \alpha_{i}$, and the statement follows.

The definition of $\bar{h}$-cores can be extended to $\mathrm{DP}_{h}$ by deciding that if $\lambda$ has repeated parts, its $\bar{h}$-core is equal to that of the partition obtained by removing those repeated 
parts. Then it is clear that if $|\lambda\rangle$ and $|\mu\rangle$ have the same $U_{q}\left(A_{2 n}^{(2)}\right)$-weight, the two partitions $\lambda$ and $\mu$ have the same $\bar{h}$-core. It follows, since $G(\mu)$ is obviously a weight vector, that its expansion on the basis $|\lambda\rangle$ involves only partitions $\lambda$ with the same $\bar{h}$-core as $\mu$.

Summarizing the discussion, we have:

Theorem 4.1. For $\mu \in \mathrm{DPR}_{h}(m)$, define $d_{\lambda \mu}(q)$ by $G(\mu)=\sum_{\lambda \in \mathrm{DP}_{h}(m)} d_{\lambda \mu}(q)|\lambda\rangle$. Then,

(i) $d_{\lambda \mu}(q) \in \mathbf{Z}[q]$

(ii) $d_{\lambda \mu}(q)=0$ unless $\lambda \unrhd \mu$, and $d_{\mu \mu}(q)=1$,

(iii) $d_{\lambda \mu}(q)=0$ unless $\lambda$ and $\mu$ have the same $\bar{h}$-core.

\section{The reduction $q=1$}

As observed by Kashiwara et al. [24], to recover the classical Fock space representation $\mathcal{F}$ of $A_{2 n}^{(2)}$, one has to introduce the inner product on $\mathcal{F}_{q}$ for which the vectors $|\lambda\rangle$ are orthogonal and the adjoint operators of the Chevalley generators are

$$
f_{i}^{\dagger}=q_{i} e_{i} t_{i}, \quad e_{i}^{\dagger}=q_{i} f_{i} t_{i}^{-1}, \quad t_{i}^{\dagger}=t_{i} .
$$

It can be checked that, for $\lambda \in \mathrm{DP}_{h}$,

$$
\langle\lambda \mid \lambda\rangle=\prod_{k>0} \prod_{i=1}^{m_{k h}}\left(1-\left(-q^{2}\right)^{i}\right),
$$

where $m_{k h}$ is the multiplicity of the part $k h$ in $\lambda$.

Let $\mathcal{F}_{1}$ denote the $A_{2 n}^{(2)}$-module obtained by specializing $q$ to 1 as in [24]. This space is strictly larger than the classical Fock space $\mathcal{F}$, since the dimension of its $m$ th homogeneous component (in the principal gradation) is $\left|\mathrm{DP}_{h}(m)\right|$ whereas that of $\mathcal{F}$ is only $|\mathrm{DP}(m)|$. Let $\mathcal{N}=\mathcal{F}_{1}^{\perp}$ denote the nullspace. It follows from (31) that $\mathcal{N}$ is a $A_{2 n}^{(2)}$-module, and from (32) that $\mathcal{N}$ is the subspace of $\mathcal{F}_{1}$ spanned by the wedge products $|\lambda\rangle$ labelled by $\lambda \in \mathrm{DP}_{h}-\mathrm{DP}$. Therefore $\mathcal{F}_{1} / \mathcal{N}$ is a $A_{2 n}^{(2)}$-module that can be identified with $\mathcal{F}$.

In this identification one has, for $\lambda=\left(\lambda_{1}, \ldots, \lambda_{r}\right) \in \mathrm{DP}$,

$$
P_{\lambda}=2^{\sum_{i=1}^{r}\left\lfloor\left(\lambda_{i}-1\right) / h\right\rfloor}|\lambda\rangle .
$$

The power of 2 comes from the fact that if $\lambda_{i}=k h$ for $k>0$, and $\nu$ denotes the partition obtained from $\lambda$ by replacing $\lambda_{i}$ by $\nu_{i}=\lambda_{i}+1$, then it follows from (11), (2) that $f_{n} P_{\lambda}$ contains $P_{\nu}$ with coefficient 1 , while $f_{n}|\lambda\rangle$ contains $|\nu\rangle$ with coefficient 2 by (8). For later use we set

$$
a_{h}(\lambda)=\sum_{i=1}^{r}\left\lfloor\frac{\lambda_{i}-1}{h}\right\rfloor .
$$




\section{Modular representations of $\widehat{\mathrm{S}}_{m}$}

We refer the reader to [7] for an up-to-date review of the representation theory of the spin symmetric groups and their combinatorics.

Let $\widehat{\mathrm{S}}_{m}$ be the spin symmetric group as defined by Schur [35], that is, the group of order $2 m$ ! with generators $z, s_{1}, \ldots, s_{m-1}$ and relations $z^{2}=1, z s_{i}=s_{i} z, s_{i}^{2}=z$, $(1 \leq i \leq m-1), s_{i} s_{j}=z s_{j} s_{i}(|i-j| \geq 2)$ and $\left(s_{i} s_{i+1}\right)^{3}=z(1 \leq i \leq m-2)$.

On an irreducible representation of $\widehat{\mathrm{S}}_{m}$, the central element $z$ has to act by +1 or by -1 . The representations for which $z=1$ are actually linear representations of the symmetric group $\mathrm{S}_{m}$, and those with $z=-1$, called spin representations correspond to two-valued representations of $\mathrm{S}_{m}$. The irreducible spin representations over a field of characteristic 0 are labelled, up to association, by strict partitions $\lambda \in \operatorname{DP}(m)$. More precisely, let $\mathrm{DP}_{+}(m)$ (resp. $\mathrm{DP}_{-}(m)$ ) be the set of strict partitions of $m$ having an even (resp. odd) number of even parts. Then, to each $\lambda \in \mathrm{DP}_{+}(m)$ corresponds a self-associate irreducible spin character $\langle\lambda\rangle$, and to each $\lambda \in \mathrm{DP}_{-}(m)$ a pair of associate irreducible spin characters denoted by $\langle\lambda\rangle$ and $\langle\lambda\rangle^{\prime}$.

According to Schur [35], the values $\langle\lambda\rangle(\rho)$ of the spin character $\langle\lambda\rangle$ on conjugacy classes of cycle-type $\rho=\left(1^{m_{1}}, 3^{m_{3}}, \ldots\right)$ are given by the expansion of the symmetric function $P_{\lambda}$ on the basis of power sums, namely

$$
P_{\lambda}=\sum_{\rho} 2^{\lceil(\ell(\rho)-\ell(\lambda)) / 2\rceil}\langle\lambda\rangle(\rho) \frac{p_{\rho}}{z_{\rho}}
$$

where $z_{\rho}=\prod_{j} j^{m_{j}} m_{j}$ ! and $\ell(\lambda)$ stands for the length of $\lambda$, that is the number of parts of $\lambda$.

For $\lambda \in \operatorname{DP}(m)$, one introduces the self-associate spin character

$$
\langle\widehat{\lambda}\rangle= \begin{cases}\langle\lambda\rangle & \text { if } \lambda \in \mathrm{DP}_{+}(m), \\ \langle\lambda\rangle+\langle\lambda\rangle^{\prime} & \text { if } \lambda \in \mathrm{DP}_{-}(m) .\end{cases}
$$

The branching theorem for spin characters of Morris [30] implies that if $\langle\hat{\lambda}\rangle$ gets identified with a weight vector of $\mathcal{F}$ by setting

$$
P_{\lambda}=2^{\lfloor(m-\ell(\lambda)) / 2\rfloor}\langle\hat{\lambda}\rangle
$$

then the $b_{\infty}$-operator $f=\sum_{i \geq 0} f_{i}^{\infty}$ implements the induction of self-associate spin characters from $\widehat{\mathrm{S}}_{m}$ to $\widehat{\mathrm{S}}_{m+1}$. Similarly, $e=e_{0}^{\infty}+2 \sum_{i>0} e_{i}^{\infty}$ implements the restriction from $\widehat{\mathrm{S}}_{m}$ to $\widehat{\mathrm{S}}_{m-1}$. Thus, the Fock space representation of $b_{\infty}$ may be viewed as the sum $\mathcal{F}=\oplus_{m} \mathcal{C}(m)$ of additive groups generated by self-associate spin characters of $\widehat{\mathrm{S}}_{m}$ in characteristic 0 . In this setting, the Chevalley generators of $b_{\infty}$ act as refined induction and restriction operators.

Now, similarly to the case $A_{n-1}^{(1)}$, the reduction from $b_{\infty}$ to $A_{2 n}^{(2)}$ parallels the reduction modulo $p=h=2 n+1$ of representations of $\widehat{\mathrm{S}}_{m}$ (from now on we assume that $h$ is an odd prime). 
More precisely, using (11) (2) (37), one sees immediately that the Chevalley generators $f_{i}$ of $A_{2 n}^{(2)}$ act on $\langle\hat{\lambda}\rangle$ as the $(r, \bar{r})$-induction operators of Morris and Yaseen $(r=n+1-i)$ [32. Hence the vectors of degree $m$ of $V\left(\Lambda_{n}\right)=U\left(A_{2 n}^{(2)}\right)^{-}|0\rangle$ can be identified with linear combinations of self-associate spin characters obtained by a sequence of $(r, \bar{r})$-inductions. It is known from modular representation theory that the maximal number of linearly independent self-associate projective spin characters of $\widehat{\mathrm{S}}_{m}$ in characteristic $p$ is equal to the number of partitions of $m$ into odd summands prime to $p$. Therefore the following result follows at once from (5).

Theorem 6.1. The self-associate projective spin characters of $\widehat{S}_{m}$ in characteristic $p$ are linear combinations of characters obtained by a sequence of $(r, \bar{r})$-inductions.

This was proved by Bessenrodt et al. for $p=3$ [8] and Andrews et al. for $p=5$ [1], but the question remained open for $p \geq 7$ [7].

Moreover, the construction of Section 4 gives an explicit basis for the space spanned by such characters. Denote by $\underline{A}(\mu)$ the column vector obtained from $A(\mu)$ by reduction $q=1$ and expansion on the basis $\langle\hat{\lambda}\rangle$. Then, $\underline{A}(\mu)$ is a projective character by (25) and $\left\{\underline{A}(\mu) \mid \mu \in \mathrm{DPR}_{p}(m)\right\}$ is a basis of the $\mathrm{Q}$-vector space of self-associate projective spin characters of $\widehat{S}_{m}$ in characteristic $p$.

These observations and the results of [26, 3, 15, 28] lead us to formulate a conjecture relating the global basis of $V\left(\Lambda_{n}\right)$ and the decomposition matrices for spin characters of the groups $\widehat{\mathrm{S}}_{m}$.

Let $\mu \in \operatorname{DPR}_{p}(m)$ and let $\underline{G}(\mu)$ stand for the image of the global basis $G(\mu)$ in $\mathcal{F}=\mathcal{F}_{1} / \mathcal{N}$, that is,

$$
\underline{G}(\mu)=\sum_{\lambda \in \operatorname{DP}(m)} 2^{b(\lambda)-a_{p}(\lambda)} d_{\lambda \mu}(1)\langle\widehat{\lambda}\rangle,
$$

where $a_{p}(\lambda)$ is given by $(34)$ and

$$
b(\lambda)=\left\lfloor\frac{m-\ell(\lambda)}{2}\right\rfloor .
$$

Then denote by $\underline{\underline{G}}(\mu)$ the vector obtained by factoring out the largest power of 2 dividing the coefficients of $\underline{G}(\mu)$ on the basis $\langle\hat{\lambda}\rangle$. For simplicity of notation, we shall identify $\underline{\underline{G}}(\mu)$ with the column vector of its coordinates on $\langle\hat{\lambda}\rangle$.

Finally, let us call reduced decomposition matrix of $\widehat{S}_{m}$ in characteristic $p$ the matrix obtained from the usual decomposition matrix for spin characters by adding up pairs of associate columns and expanding the column vectors so obtained on the basis $\langle\widehat{\lambda}\rangle$. This is a matrix with $|\mathrm{DP}(m)|$ rows and $\left|\mathrm{DPR}_{p}(m)\right|$ columns. The definition is illustrated in Table 2 and Table 3. (Table 2 is taken from [32], except for the column labels which are ours and will be explained in the next section.)

Conjecture 6.2. (i) The set of column vectors of the reduced decomposition matrix of $\widehat{\mathrm{S}}_{m}$ in odd characteristic $p$ such that $p^{2}>m$ coincides with $\left\{\underline{\underline{G}}(\mu) \mid \mu \in \operatorname{DPR}_{p}(m)\right\}$. 
Table 2. The decomposition matrix of $\widehat{\mathrm{S}}_{10}$ in characteristic 3 .

\begin{tabular}{llllllll}
\hline & $(3331)$ & $(3331)^{\prime}$ & $(4321)$ & $(4321)^{\prime}$ & $(532)$ & $(541)$ & $(541)^{\prime}$ \\
\hline$\langle 4321\rangle$ & 0 & 0 & 1 & 1 & 0 & 0 & 0 \\
$\langle 532\rangle$ & 0 & 0 & 0 & 0 & 1 & 0 & 0 \\
$\langle 532\rangle^{\prime}$ & 0 & 0 & 0 & 0 & 1 & 0 & 0 \\
$\langle 541\rangle$ & 1 & 1 & 1 & 1 & 0 & 0 & 1 \\
$\langle 541\rangle^{\prime}$ & 1 & 1 & 1 & 1 & 0 & 1 & 0 \\
$\langle 631\rangle$ & 2 & 2 & 1 & 1 & 0 & 1 & 1 \\
$\langle 631\rangle^{\prime}$ & 2 & 2 & 1 & 1 & 0 & 1 & 1 \\
$\langle 64\rangle$ & 1 & 1 & 0 & 0 & 0 & 1 & 1 \\
$\langle 721\rangle$ & 1 & 1 & 0 & 1 & 0 & 0 & 1 \\
$\langle 721\rangle^{\prime}$ & 1 & 1 & 1 & 0 & 0 & 1 & 0 \\
$\langle 73\rangle$ & 1 & 1 & 1 & 1 & 0 & 1 & 1 \\
$\langle 82\rangle$ & 0 & 0 & 0 & 0 & 1 & 0 & 0 \\
$\langle 91\rangle$ & 1 & 1 & 1 & 1 & 0 & 0 & 0 \\
$\langle 10\rangle$ & 0 & 1 & 0 & 0 & 0 & 0 & 0 \\
$\langle 10\rangle^{\prime}$ & 1 & 0 & 0 & 0 & 0 & 0 & 0 \\
\hline
\end{tabular}

Table 3. The reduced decomposition matrix of $\widehat{\mathrm{S}}_{10}$ in characteristic 3 .

\begin{tabular}{|c|c|c|c|c|}
\hline & $(3331)$ & $(4321)$ & $(532)$ & (541) \\
\hline$\langle\widehat{4321}\rangle$ & 0 & 2 & 0 & 0 \\
\hline$\langle\widehat{532}\rangle$ & 0 & 0 & 1 & 0 \\
\hline$\langle\widehat{541}\rangle$ & 2 & 2 & 0 & 1 \\
\hline$\langle 631\rangle$ & 4 & 2 & 0 & 2 \\
\hline$\langle\widehat{64}\rangle$ & 2 & 0 & 0 & 2 \\
\hline$\langle\widehat{721}\rangle$ & 2 & 1 & 0 & 1 \\
\hline$\langle\widehat{73}\rangle$ & 2 & 2 & 0 & 2 \\
\hline$\langle 82\rangle$ & 0 & 0 & 1 & 0 \\
\hline$\langle\widehat{91}\rangle$ & 2 & 2 & 0 & 0 \\
\hline$\langle\widehat{10}\rangle$ & 1 & 0 & 0 & 0 \\
\hline
\end{tabular}

(ii) For $p^{2} \leq m$, the reduced decomposition matrix of $\widehat{\mathrm{S}}_{m}$ is obtained by postmultiplying the matrix whose columns are $\underline{G}(\mu)$ by a unitriangular matrix with nonnegative entries.

Our conjecture has been checked on the numerical tables computed by Morris and Yaseen $(p=3)$ [32] and Yaseen $(p=5,7,11)$ [39]. Thus, for $p=3, m=11$, the columns of the reduced decomposition matrix are

$$
\underline{\underline{G}}(3332), \underline{\underline{G}}(4331)+\underline{\underline{G}}(641), \underline{\underline{G}}(5321), \underline{\underline{G}}(542), \underline{\underline{G}}(641) .
$$




\section{Labels for irreducible modular spin characters and partition identities}

The labels for irreducible modular representations of symmetric groups form a subset of the ordinary labels [18]. It is therefore natural to look for a labelling scheme for irreducible modular spin representations of $\widehat{S}_{m}$ using a subset of $\operatorname{DP}(m)$. This was accomplished for $p=3$ by Bessenrodt et al. [8], who found that the Schur regular partitions of $m$ form a convenient system of labels. These are the partitions $\lambda=\left(\lambda_{1}, \ldots, \lambda_{r}\right)$ such that $\lambda_{i}-\lambda_{i+1} \geq 3$ for $i=1, \ldots, r-1$, and $\lambda_{i}-\lambda_{i+1}>3$ whenever $\lambda_{i} \equiv 0 \bmod 3$.

In [8], it was also conjectured that for $p=5$, the labels should be the partitions $\lambda=\left(\lambda_{1}, \ldots, \lambda_{r}\right)$ satisfying the following conditions: (1) $\lambda_{i}>\lambda_{i+1}$ for $i \leq r-1$, (2) $\lambda_{i}-\lambda_{i+2} \geq 5$ for $i \leq r-2$, (3) $\lambda_{i}-\lambda_{i+2}>5$ if $\lambda_{i} \equiv 0 \bmod 5$ or if $\lambda_{i}+\lambda_{i+1} \equiv 0 \bmod 5$ for $i \leq r-2$, and (4) there are no subsequences of the following types (for some $j \geq 0$ ): $(5 j+3,5 j+2),(5 j+6,5 j+4,5 j),(5 j+5,5 j+1,5 j-1),(5 j+6,5 j+5,5 j, 5 j-1)$. This conjecture turned out to be equivalent to a $q$-series identity conjectured long ago by Andrews in the context of extensions of the Rogers-Ramanujan identities, and was eventually proved by Andrews et al. [1]. The authors of [1] observed however that such a labelling scheme could not be extended to $p=7,11,13$ (see also [7]).

In terms of canonical bases, the obstruction can be understood as follows. Assuming our conjecture and using the results of [8, 1], one can see that for $p=3,5$, the labels of [8] and [1] are exactly the partitions indexing the lowest nonzero entries in the columns of the matrices $D_{m}(q)=\left[d_{\lambda \mu}(q)\right]_{\lambda, \mu \vdash m}$. For example, in Table 1, these are (10), (91), (82) and (73), which are indeed the Schur regular partitions of 10 . The problem is that for $p \geq 7$, it can happen that two columns have the same partition indexing the lowest nonzero entry. For example, with $p=7(n=3)$ and $m=21$, the two canonical basis vectors

$G(75432)=|75432\rangle+q^{2}|76431\rangle+q|7752\rangle+q^{3}|7761\rangle+q^{2}|8643\rangle+\left(q^{2}+q^{4}\right)|8652\rangle+$ $q^{3}|876\rangle+q^{4}|9543\rangle+\left(q^{4}+q^{6}\right)|9651\rangle+q^{5}|975\rangle$

and

$G(654321)=|654321\rangle+q|75432\rangle+q|76431\rangle+q|76521\rangle+q^{2}|7743\rangle+q^{2}|7752\rangle+$

$q^{2}|7761\rangle+q^{3}|777\rangle+\left(q^{3}+q^{5}\right)|8643\rangle+\left(q^{3}+q^{5}\right)|8652\rangle+\left(q^{4}-q^{8}\right)|876\rangle+\left(q^{3}+q^{5}\right)|9651\rangle+$ $\left(q^{4}+q^{6}\right)|975\rangle$

have the same bottom partition (975) (compare [7], end of Section 3).

On the other hand the partitions indexing the highest nonzero entries in the columns of $D_{m}(q)$ are the labels of the crystal graph (by Theorem 4.1(ii)), so that they are necessarily distinct. Therefore, we propose to use the set

$$
\begin{aligned}
\mathrm{DPR}_{p}(m)= & \left\{\lambda=\left(\lambda_{1}, \ldots, \lambda_{r}\right) \vdash m \mid 0<\lambda_{i}-\lambda_{i+1} \leq p \text { if } \lambda_{i} \not \equiv 0 \text { mod } p,\right. \\
& \left.0 \leq \lambda_{i}-\lambda_{i+1}<p \text { if } \lambda_{i} \equiv 0 \bmod p,(1 \leq i \leq r)\right\}
\end{aligned}
$$


for labelling the irreducible spin representations of $\widehat{\mathrm{S}}_{m}$ in characteristic $p$. Indeed its definition is equally simple for all $p$. Moreover, because of Theorem 4.1(iii), this labelling would be compatible with the $p$-block structure, which can be read on the $\bar{p}$-cores. Also, it is adapted to the calculation of the vectors $\underline{A}(\mu)$ which give an approximation to the reduced decomposition matrix.

Finally, we note that since $\mathrm{DPR}_{p}$ provides the right number of labels we have the following partition identity

$$
\sum_{m \geq 0}\left|\mathrm{DPR}_{p}(m)\right| t^{m}=\prod_{\substack{i \text { odd } \\ i \neq 0 \text { mod } p}} \frac{1}{1-t^{i}}
$$

which for $p=3,5$ is a counterpart to the Schur and Andrews-Bessenrodt-Olsson identities.

This happens to be a particular case of a theorem of Andrews and Olsson [2]. Namely, one gets (40) by taking $A=\{1,2,3, \ldots, p-1\}$ and $N=p$ in Theorem 2 of [2]. A combinatorial proof of a refinement of the Andrews-Olsson partition identity has been given by Bessenrodt [6].

One can also get a direct proof of (40) without using representation theory by simply considering the bijections (21).

\section{Discussion}

We have used the level 1 -deformed Fock spaces of Kashiwara et al. to compute the canonical basis of the basic representation of $U_{q}\left(A_{2 n}^{(2)}\right)$, and we have formulated a conjectural relation with the decomposition matrices of the spin symmetric groups in odd characteristic $p=2 n+1$.

As in the case of $A_{n-1}^{(1)}$, it is reasonable to expect that in general, that is when $2 n+1$ is not required to be a prime, the canonical basis is related to a certain family of Hecke algebras at $(2 n+1)$ th roots of unity. A good candidate might be the Hecke-Clifford superalgebra introduced by Olshanski [34].

The case of $2 n$th roots of unity should then be related to the Fock space representation of the affine Lie algebras of type $D_{n+1}^{(2)}$. In particular we believe that the fact used by Benson [5] and Bessenrodt-Olsson [9] that the 2-modular irreducible characters of $\widehat{\mathrm{S}}_{m}$ can be identified with the 2-modular irreducible characters of $\mathrm{S}_{m}$ corresponds in the realm of affine Lie algebras to the isomorphism $D_{2}^{(2)} \simeq A_{1}^{(1)}$.

\section{Acknowledgements}

We thank T. Miwa and A.O. Morris for stimulating discussions, and G.E. Andrews for bringing references [2, 6] to our attention. 


\section{References}

[1] Andrews G E, Bessenrodt C and Olsson J B 1994 Trans. Amer. Math. Soc. 344597

[2] Andrews G E and Olsson J B 1991 J. reine angew. Math. 413198

[3] Ariki S 1996 On the decomposition numbers of the Hecke algebra of $G(m, 1, n)$, preprint

[4] Ariki S, Nakajima T and Yamada H-F 1995 J. Phys. A: Math. Gen. 28 L357

[5] Benson D 1987 Proc. Sympos. Pure Math. 47381

[6] Bessenrodt C 1991 Europ. J. Combinatorics 12271

[7] Bessenrodt C 1994 Séminaire Lotharingien de Combinatoire B33a, http://cartan.u-strasbg.fr:80/〜 slc/opapers/s33bess.htm]

[8] Bessenrodt C, Morris A O and Olsson J B 1994 J. Algebra 164146

[9] Bessenrodt C and Olsson J B 1993 Institut fur Experimentelle Mathematik (Essen) preprint 17

[10] Date E, Jimbo M, Kashiwara M and Miwa T 1982 Physica D 4343

[11] Date E, Jimbo M, Kashiwara M and Miwa T 1982 Publ. RIMS Kyoto Univ. 181077

[12] Foda O, Leclerc B, Okado M, Thibon J-Y and Welsh T A 1997 preprint, q-alg/9701020

[13] Foda O, Okado M and Warnaar S O 1996 J. Math. Phys. 37965

[14] Grojnowski I 1994 Internat. Math. Res. Notices 5215

[15] Grojnowski I 1995 Personal communication

[16] Hayashi T 1990 Commun. Math. Phys. 127129

[17] James G 1990 Proc. London Math. Soc. 60225

[18] James G and Kerber A 1981 The Representation Theory of the Symmetric Group (Reading Mass.: Addison-Wesley)

[19] Jarvis P and Yung C M 1994 Lett. Math. Phys. 3045

[20] Kac V G, Kazhdan D A, Lepowsky J and Wilson R L 1981 Adv. Math. 4283

[21] Kang S-J, Kashiwara M, Misra K C, Miwa T, Nakashima T and Nakayashiki A 1992 Int. J. Mod. Phys. A 7 Suppl. 1A 449

[22] Kang S-J, Kashiwara M, Misra K C, Miwa T, Nakashima T and Nakayashiki A 1992 Duke Math. J. 68499

[23] Kashiwara M 1991 Duke Math. J. 63465

[24] Kashiwara M, Miwa T, Petersen J-U H and Yung C M 1996 Selecta Math. 2415

[25] Kashiwara M, Miwa T and Stern E 1996, Selecta Math. 1787

[26] Lascoux A, Leclerc B and Thibon J-Y 1996 Commun. Math. Phys. 181205

[27] Lascoux A, Leclerc B and Thibon J-Y 1997 J. Math. Phys. 38 (2) 1041

[28] Leclerc B and Thibon J-Y 1996 Internat. Math. Res. Notices 9447

[29] Misra K C and Miwa T 1990 Commun. Math. Phys. 13479

[30] Morris A O 1965 Can. J. Math. 17543

[31] Morris A O and Yaseen A K 1986 Math. Proc. Camb. Phil. Soc. 9923

[32] Morris A O and Yaseen A K 1988 Proc. Royal Soc. Edinburgh 108A 145

[33] Nakajima T and Yamada H-F 1994 J. Phys. A: Math. Gen. 27 L171

[34] Olshanski G I 1992 Lett. Math. Phys. 2493

[35] Schur I 1911 J. Reine Ang. Math. 139155

[36] Soergel W 1997 Represent. Theory 183

[37] Soergel W 1997 Represent. Theory 1115

[38] Stern E 1995 Internat. Math. Res. Notices 4201

[39] Yaseen A K 1987 Thesis, University of Wales 
[40] You Y 1989 Adv. Ser. in Math. Phys. 7449 